\title{
Restless Legs Syndrome in Liver Transplant Recipients
}

\author{
Rose Franco $^{1}$, Syed Rizvi ${ }^{2}$, Jose Franco ${ }^{2}$, Kia Saeian ${ }^{2}$, Farhan Rizvi ${ }^{3}$ \\ ${ }^{1}$ Division of Pulmonary and Critical Care, Medical College of Wisconsin, Milwaukee, USA; ${ }^{2}$ Division of Gastroenterology and \\ Hepatology, Medical College of Wisconsin, Milwaukee, USA; ${ }^{3}$ Department of Ophthalmology, Medical College of Wisconsin, Mil- \\ waukee, USA. \\ Email: srizvi@mcw.edu
}

Received December $14^{\text {th }}, 2012$; revised January $25^{\text {th }}$, 2013; accepted February $20^{\text {th }}, 2013$

\begin{abstract}
Background: The prevalence of restless legs syndrome (RLS) is approximately $10 \%$ in general population. This condition is more prevalent in certain diseases and we previously reported the prevalence of RLS in chronic liver disease population to be $62 \%$. Objectives: Our aim was to assess the self-reported prevalence of RLS using an RLS symptom specific questionnaire in liver transplant patients. Methods: Subjects were a convenience sample in transplant clinic. They completed a validated survey for core RLS symptoms and if positive went on to completed a telephone survey using the validated International RLS Severity Scale Questionnaire (IRLS) and the Johns Hopkins RLS QoL survey to measure the effect of RLS symptoms on daily activities. Results: 40 patients surveyed, RLS was found in 16 subjects $(40 \%)$ with moderate severity $\{17$ (SD +7.2 , high score 31$)\}$. Hepatitis $C$ as indication for liver transplant was more likely associated with RLS $(p=0.05)$. Calcium channel blockers were protective $(p=0.032)$ while antidopaminergic agent use was statistically significant for RLS symptom $(\mathrm{p}=0.005)$. On multiple linear regression analysis, diabetes $(\mathrm{p}$ $=0.024)$ and use of antidepressants/antihistamines $(p=0.049)$ were associated with RLS. Quality of Life (QoL) surveys specific to RLS suggested RLS symptoms resulted in significantly diminished QoL, with an average QoL score of 80 (SD + 11.7). Conclusion: There was a very high prevalence of RLS in our liver transplant patients with majority experiencing moderate or severe symptoms. The explanation for this higher prevalence is likely due to combination of comorbidites as well as medications that can trigger RLS.
\end{abstract}

Keywords: Restless Legs Syndrome (RLS); Liver Transplant; Prevalence

\section{Background}

Restless leg syndrome (RLS) is a common clinical syndrome with significant impact on sleep quality and quality of life (QoL) and a prevalence of 5\% - 15\% [1-3] in general population.

This syndrome is most often identified by the following tetrad of symptoms: 1 ) focal akathesia (an irresistible desire to move localized to the extremities that is periodic and distressing due to abnormal sensations); 2) motor restlessness; 3) quiescagenic symptoms (engendered or exacerbated by remaining at rest); and 4) circadian phenomenon (symptoms worsen in the evening/night for a person entraining to a normal circadian rhythm).

Restless leg syndrome is strongly associated with certain conditions including diabetes [4-6], chronic kidney disease (uremic neuropathy) $[7,8]$, iron deficiency anemia [9,10], obesity [11], vitamin deficiency states related with peripheral neuropathy $[12,13]$ and folate deficiency [14].

There is little known about the natural history of secondary RLS in association with specific disease states.
We have previously reported the prevalence of RLS symptoms in our institution's specialty clinic chronic liver disease population to be $62 \%$ [15]. We undertook this current study in an attempt to ascertain any causality of chronic liver disease with RLS by studying a population with reversal of chronic liver disease, namely those with a successful liver transplant.

Prior work in the area of renal failure suggests that some RLS can be reversible. In those with chronic renal failure on hemodialysis, transplantation appears to decrease the prevalence of the condition significantly. Other transplant populations have not faired as well [16]. Minai et al. [17] and Javaheri S. et al. [18] have reported a prevalence of $47.6 \%$ and $45 \%$ respectively in lung and heart transplant recipients, there is no prevalence data for RLS in liver transplant recipients. The primary aim of this study was to determine the self-reported surveybased prevalence of RLS symptoms in patients who had undergone liver transplantation and were now followed in an outpatient liver transplant clinic. The secondary aims were to determine and compare the patient and laboratory characteristics of this population with or 
without RLS and for those identified with RLS to measure its severity and impact on QoL through use of validated tools developed by the International Restless Legs Syndrome Study Group (IRLSSG) [19,20].

\section{Methods}

\subsection{Subjects}

All Liver transplant recipients seen in the outpatient liver transplant clinic between January 2008 to April 2008 were invited to participate in the study. This was a convenience sample. The patient population was heterogeneous in age, gender and etiology of their primary liver disease. All of these patients were routinely followed by the liver transplant team at our institution and were clinically stable with stable graft function on standard immunosuppression and prophylactic antibiotics based on our institution's liver transplant protocol. Those with comorbid diseases known to be associated with RLS were not excluded from participation.

\subsection{Study Protocol}

The protocol for this study was approved by the institutional review board of the Medical College of Wisconsin and conforms to the ethical guidelines of the 1975 Declaration of Helsinki. Consent to participate was obtained at the time of the scheduled Transplant clinic visit by the treating hepatologist. The subjects were asked if they would be willing to fill out a one-page survey and to be contacted by phone for follow-up questions if necessary. The content of the survey was not revealed before consent to participate was obtained to reduce selection bias. An initial screening survey was a self-response questionnaire during an already scheduled clinic visit. The survey queried demographic and health information including recognized conditions associated with RLS and 5 RLS qualitative screening questions based on IRLSSG and the National Institutes of Health definition of the syndrome.

Survey results were reviewed by the investigators and a trained investigator did phone follow-up with individual participants who were positive for RLS was utilized to confirm the RLS symptoms reported on the survey. The investigator again queried and confirmed the previous responses from the subjects reported on their survey. Once RLS was confirmed, the investigator also performed the International RLS Severity Scale Questionnaire (IRLS) with the subjects. This scale is a validated rating scale of severity based on the discomfort due to RLS, the need to move the legs, the severity of sleep disturbances, the impact of RLS on daily activity and the overall severity of the symptoms and the relief of discomfort by movement. The participants identified with
RLS also completed an RLS specific QoL survey; the Johns Hopkins RLS QoL survey [21]. This survey measures the effect of RLS symptoms in the context of their impact on performing activities such as occupational and social responsibilities, and activities of daily living.

A standardized chart review was also conducted on all subjects. Items audited included date of transplant, liver function tests, renal function, hemoglobin, ferritin, previous or current alcohol use and additional diagnoses such as anemia, neuropathy, diabetes peripheral neuropathy, chronic kidney disease and medications known to precipitate RLS.

\section{Statistical Analysis}

Sample size for 2 proportions using a power value of 0.8 and assumed post-transplant rate of $30 \%$ was performed using Minitab software and resulted in a sample size of 40. All additional statistical analysis was performed using Sigma Plot. Descriptive statistics for variables such as laboratory values were performed and represented as means with 95\% confidence of interval (CI). Fisher's exact test and Pearson's chi square test used for medications and other categorical measures. Mann-Whitney was performed for certain measures where it was appropriate. ANOVA on Rank and Dunnett test was performed on group means for identifying any variable associated with RLS. A multiple linear regression analysis was also performed.

\section{Results}

All liver transplant recipients seen in clinic between January 2008 and April 2008 were invited to participate. Forty three patients initially agreed to participate in the study between January 2008 and April 2008. Two patients did not complete the survey. One patient subsequently requested to be withdrawn from the study and that survey and analysis were not included in the study. The demographics of the forty subjects analyzed were $54.7 \pm 1.8$ (mean \pm SEM) years of age (Table 1) and there were 27 (67.5\%) men and 13 (32.5\%) women. The reason for transplant in the overall group included 10 (25\%) for alcoholic cirrhosis, 10 (25\%) for hepatitis C, 4 (10\%) for PSC, 5 (12.5\%) for NASH, 1 (2.5\%) for Hepatitis B, 1 (2.5\%) for Hepatitis B/C co-infection, 1 (2.5\%) for Budd chiari syndrome, 1 (2.5\%) for cryptogenic cirrhosis, 2 (5\%) for primary biliary cirrhosis, 1 (2.5\%) for hereditary hemochromatosis and $4(10 \%)$ subjects for liver failure due to autoimmune hepatitis. In the overall group, time since transplant was $6.7 \pm 0.65$ years (mean \pm SEM). Liver function tests were mildly elevated in only one patient in RLS group and in five patients in non-RLS group however out of those five patients only two had ALT values more than twice the upper limit of 
Table 1. Descriptive summary of the transplant recipients.

\begin{tabular}{|c|c|c|c|}
\hline Factor & RLS (16) & N-RLS (24) & Overall value (40) \\
\hline \multicolumn{4}{|l|}{ Age (Mean, SEM) } \\
\hline & $56.9 \pm 2.49$ & $53.2 \pm 2.72$ & $54.7 \pm 1.8$ \\
\hline \multicolumn{4}{|l|}{ Gender (N, \%) } \\
\hline Male & 11 & 16 & $27(67.5)$ \\
\hline Female & 5 & 8 & $13(32.5)$ \\
\hline \multicolumn{4}{|l|}{ Time from transplant (y) (Mean, SEM) } \\
\hline & $6.5 \pm 1.2$ & $6.8 \pm 0.76$ & $6.7 \pm 0.65$ \\
\hline \multicolumn{4}{|l|}{ Reason for transplant $(\mathrm{N}, \%)$} \\
\hline Primary sclerosing cholangitis & 2 & 2 & $4(10)$ \\
\hline Alcohol & 2 & 8 & $10(25)$ \\
\hline Hepatitis C & 7 & 3 & $10(25)$ \\
\hline Hepatitis B & 1 & 0 & $1(2.5)$ \\
\hline Hepatitis B/C co-infection & 1 & 0 & $1(2.5)$ \\
\hline Budd Chiari syndrom & 0 & 1 & $1(2.5)$ \\
\hline Cryptogenic cirrhosis & 0 & 1 & $1(2.5)$ \\
\hline Primary biliary cirrhosis & 0 & 2 & $2(5)$ \\
\hline Hereditary hemochromatosis & 0 & 1 & $1(2.5)$ \\
\hline Non-alcoholic Steatohepatitis & 2 & 3 & $5(12.5)$ \\
\hline Autoimmune hepatitis & 1 & 3 & $4(10)$ \\
\hline \multicolumn{4}{|l|}{ Comorbidities (N, \%) } \\
\hline Diabetes Mellitus & $10(62.5)$ & $10(41.6)$ & $20(50)$ \\
\hline Neuropathy & $5(31.25)$ & $2(8.33)$ & $7(17.5)$ \\
\hline Chronic Kidney Disease (GFR < 60) & $10(62.5)$ & $16(66.6)$ & $26(65)$ \\
\hline \multicolumn{4}{|l|}{ Labs (Mean, SEM) } \\
\hline Hemoglobin & $13.5 \pm 0.4$ & $13.49 \pm 0.38$ & $13.5 \pm 0.26$ \\
\hline Calcium & $9.2 \pm 0.06$ & $9.2 \pm 0.07$ & $9.2 \pm 0.0 .05$ \\
\hline Creatinine & $1.46 \pm 0.12$ & $1.35 \pm 0.05$ & $1.39 \pm 0.06$ \\
\hline Magnesium & $1.75 \pm 0.05$ & $1.8 \pm 0.04$ & $1.77 \pm 0.03$ \\
\hline \multicolumn{4}{|l|}{ Medications (N, \%) } \\
\hline Prednisone & $4(25)$ & $5(20.8)$ & $9(22.5)$ \\
\hline Cyclosporine & $2(12.5)$ & $1(4.1)$ & $3(7.5)$ \\
\hline Tacrolimus & $11(68.7)$ & $14(58.3)$ & $25(62.5)$ \\
\hline Mychophenolate & $5(31.2)$ & $6(25)$ & $11(27.5)$ \\
\hline Sirolimus & $1(6.25)$ & $8(33.3)$ & $9(22.5)$ \\
\hline Calcium Channel blockers & $1(6.25)$ & $9(37.5)$ & $10(25)$ \\
\hline Antidepressants & $4(25)$ & $1(4.1)$ & $5(12.5)$ \\
\hline Benzodiazepines & $0(0)$ & $2(8.3)$ & $2(5)$ \\
\hline Antihistamines & $5(31.25)$ & $2(8.3)$ & $7(17.5)$ \\
\hline
\end{tabular}


normal. None of the patients had evidence or suspicion for graft failure at the time of recruitment.

Of the 40 subjects, 16 reported RLS symptoms on the validated survey which was later confirmed by physician telephone interview. No subject contact was found to have a false positive RLS survey. In our clinic sample, the prevalence of RLS was $40 \%$ (CI 26\% - 57\%) (p < $0.05 \%$ ) which is significantly elevated compared to general population survey based prevalence. There were twice as many men as women in both groups. Comparison between those with RLS and those without revealed a similarly high rate of diabetes in both groups (Table 1). Use of medications (calcium channel blockers, antidepressants, dopaminergic antagonists) known to cause or exacerbate RLS was low in both groups. Four of sixteen patients (25\%) reporting RLS were on antidepressants known to cause RLS vs. one patient in non-RLS group (4.1\%). Five subjects in RLS group (31.25\%) and two in non-RLS group (8.33\%) were on antihistamines. Medications used to treat RLS such as benzodiazepine were used in two patients in non-RLS group however no patient with RLS was on benzodiazepines. No subject in either group was on a dopaminergic agent.

There was no active alcohol use reported by any patient in either group. Folic acid levels, vitamin B12 levels and iron panel and ferritin were available for very few patients in either group and analysis was not done on those factors.

A comparison of RLS and non-RLS groups was made to evaluate for variables in both groups. There was no statistical difference in various immunosuppressants used in both RLS and non-RLS group (Table 2). In regards to etiology of liver disease for liver transplant and its association with the presence of RLS, in those with hepatitis $\mathrm{C}$ there was a trend towards statistical significance in RLS patients $(p=0.05)$ but alcohol related liver disease was not statistically significant $(p=0.263)$. All other etiologies for liver transplantation were too small in number for analysis. Medications influencing RLS such as use of calcium channel blockers, antihistamines, benzodiazepines, antidepressants were analyzed and use of calcium channel blockers appeared protective against RLS (0.032) however use of an antidopaminergic agent (antidepressants, antihistamines) was statistically significant for those with RLS (0.005). In regards to lab values, a GFR with cut off value of $>50 \mathrm{ml} /$ minute $/ \mathrm{m}^{2}$ was not statistically significant $(0.755)$. To further evaluate the factors known to influence the RLS a multiple linear regression analysis was done for GFR (cutoff > 50) (renal failure), diabetes, neuropathy, calcium channel blockers, hemoglobin (for anemia), serum magnesium, serum calcium, antidepressants/antihistamines and etiology of liver disease. The presence of Diabetes (0.024) and use of antidepressants/antihistamines (0.049) were found to be as- sociated with RLS. Use of calcium channel blockers again showed to be protective against RLS (0.013).

After reviewing the charts of each patient reporting RLS and excluding all known risk factors for secondary RLS, (iron deficiency anemia, diabetes, kidney disease, neuropathy, meds with dopaminergic effects, anticholinergic effect and antihistamines) 2 out of 16 patients with RLS (12.5\%) were found to have no clear causative factor to result in RLS. While metabolic profiles were not complete, of those with RLS who had a TSH checked, none were abnormal.

\section{RLS Quality of Life and Severity Assessment}

Twelve of 16 subjects reporting RLS symptoms completed and returned the RLS QoL survey. The mean RLSQoL score was 80 (SD + 11.7). This score reflects moderately diminished QoL based on previous validation with a general population surveyed. A 10 question survey to assess the severity of RLS symptoms (IRLS) was also completed by these 12 RLS positive patients. The mean score was 17 (SD + 7.2, high score 31) indicating moderate severity of RLS when compared to the validated and published rating scale for this tool (IRLSSG).

\section{Discussion}

This is the first study to report the prevalence and factors associated with RLS in liver transplant recipients. In this study of our institution's transplant clinic population, we found a $40 \%$ prevalence which is significantly higher than that reported in studies in general population (5\% $15 \%)$ [1-3]. This finding however is similar to the rates reported for lung and heart transplant recipients $[17,18]$ and is also in the range of what we have previously reported for patients with chronic liver disease [15]. However, in contrast to our previous study on patients with chronic liver disease [15], post-transplant patients have excellent graft function with no evidence of liver failure in any subject. Also there is a difference in medication profile in the two groups of patients and majority of the patients in later group are considered cured with no evidence of recurrent liver disease except in patients who have recurrent hepatitis $C$ with no evidence of cirrhosis. This study was aimed at patients whose liver disease was resolved after liver transplantation and had functioning hepatic grafts at the time of study. Hence one possible explanation for this higher prevalence is likely due to combination of comorbidities as well as medications that can trigger RLS. Group comparisons of liver transplant recipients with and without RLS at our center does not provide us with any clear mechanism however on multivariate analysis diabetes which is a known risk factor was statistically significant in RLS group as well as use 
Table 2. Comparison of RLS and non-RLS groups for several categorical measures.

\begin{tabular}{|c|c|c|c|c|c|}
\hline \multirow{2}{*}{ Drugs } & \multicolumn{2}{|c|}{ RLS } & \multicolumn{2}{|c|}{ No RLS } & \multirow{2}{*}{ p-value } \\
\hline & $\mathrm{N}$ & $(\%)$ & $\mathrm{N}$ & (\%) & \\
\hline \multicolumn{6}{|l|}{ Cyclosporine } \\
\hline Yes & 2 & 12.5 & 1 & 4.16 & \multirow{2}{*}{$0.553^{*}$} \\
\hline No & 14 & 87.5 & 23 & 95.83 & \\
\hline \multicolumn{6}{|l|}{ Mycophenolate } \\
\hline Yes & 4 & 25 & 7 & 29.1 & \multirow{2}{*}{$0.942^{*}$} \\
\hline No & 12 & 75 & 17 & 70.8 & \\
\hline \multicolumn{6}{|l|}{ Sicrolimus } \\
\hline Yes & 1 & 6.25 & 8 & 33.3 & \multirow{2}{*}{$0.061^{*}$} \\
\hline No & 15 & 93.75 & 16 & 66.6 & \\
\hline \multicolumn{6}{|l|}{ Tacrolimus } \\
\hline Yes & 12 & 75 & 13 & 54.1 & \multirow{2}{*}{$0.318^{*}$} \\
\hline No & 4 & 25 & 11 & 45.9 & \\
\hline \multicolumn{6}{|c|}{ (Amlodipine/Diltiazem) } \\
\hline Yes & 1 & 6.25 & 9 & 37.5 & \multirow{2}{*}{0.032} \\
\hline No & 15 & 93.75 & 15 & 62.5 & \\
\hline \multicolumn{6}{|l|}{ Factor } \\
\hline \multicolumn{6}{|l|}{ Creatinine } \\
\hline$>1.5$ & 6 & 37.5 & 8 & 33.3 & \multirow{2}{*}{$0.529^{*}$} \\
\hline$<1.5$ & 10 & 62.5 & 16 & 66.6 & \\
\hline \multicolumn{6}{|l|}{ Diabetes } \\
\hline Yes & 10 & 62.5 & 10 & 41.6 & \multirow{2}{*}{$0.3^{*}$} \\
\hline No & 6 & 37.5 & 14 & 58.3 & \\
\hline \multicolumn{6}{|l|}{ Neuropathy } \\
\hline Yes & 4 & 25 & 3 & 12.5 & \multirow{2}{*}{$0.407^{*}$} \\
\hline No & 12 & 75 & 21 & 87.5 & \\
\hline \multicolumn{6}{|l|}{ Narcotics } \\
\hline Yes & 4 & 25 & 6 & 25 & \multirow{2}{*}{$1.000^{*}$} \\
\hline No & 12 & 75 & 18 & 75 & \\
\hline \multicolumn{6}{|l|}{ Hep C } \\
\hline Yes & 7 & 43.75 & 3 & 12.5 & \multirow{2}{*}{$0.05^{*}$} \\
\hline No & 9 & 56.25 & 21 & 87.5 & \\
\hline Alcohol & & & & & \\
\hline Yes & 2 & 12.5 & 8 & 33.34 & 0.263 \\
\hline No & 14 & 87.5 & 16 & 66.66 & 0.205 \\
\hline GFR $>50$ & & & & & \\
\hline & 7 & 43.75 & 12 & 50 & $0.755^{*}$ \\
\hline & 9 & 56.25 & 12 & 50 & . \\
\hline Antidepressants/ & & & & & \\
\hline & 9 & & 3 & & $0.005($ Sig) \\
\hline & 7 & & 21 & & \\
\hline
\end{tabular}

"Fisher exact/chi square test; ${ }^{* *}$ Mann-Whitney test. 
of antihistamines/antidepressants was associated with RLS. Interestingly, the presence of hepatitis $C$ showed a trend toward statistical significance for RLS on Fisher exact test and not significant by multiple regression analysis. Population studies of RLS have indicated that RLS has a female predominance 20. In our study however, there were more male participants in general but there was a 2 to 1 male predominance in both RLS and nonRLS groups. This finding may very well be due to higher percentage of male liver transplant recipients in general (UNOS.org). While renal failure has been linked to a higher risk of RLS [22,23], in our study a decreased GFR did not show influence on occurrence of RLS.

A number of medications are believed to either induce or exacerbate the symptoms of RLS. These include selective serotonin re-uptake inhibitors, tricyclic anti-depressants [24-27], non-opioid analgesics, lithium [28], beta-blockers, histamine-2 blockers, dopamine antagonist and ethanol [29]. There was a significantly higher use of these medications in the RLS group compared with the non-RLS group (Table 2). Alcohol use has been related to neuropathy which could be associated with RLS however most of the patients transplanted for alcoholic cirrhosis in our cohort were in the non-RLS group. The finding of a trend toward significant associated between RLS and Hepatitis C is interesting. Although no clear link has been documented between the two, chronic hepatitis C is associated with neuropathy [30,31] and neuropathy can be associated or mistaken for RLS. Whether hepatitis C is the cause for RLS in some of these patients warrants further study. Although calcium channel blockers are known to cause the RLS, this was not the case in our cohort of patients however the likely explanation to this could be a sampling bias.

The limitations of our study include its relatively small size, use of convenience sample, single center experience, inability to control for time from transplant and lack of data on pre-existing RLS and lab values (e.g. iron panel) for all patients. We were further limited by the retrospective nature of the chart review portion and the lack of corroboration of the questionnaire-based findings with direct physical examination and laboratory testing specifically targeted to RLS risk factors.

Future directions of study should be a prospective analysis of complete blood profiles including vitamin B12 and other nutritional markers, CMV status, and detailed physical examinations coupled with nerve conduction studies to confirm that neuropathic pain is not being misinterpreted as RLS symptoms.

In conclusion, RLS symptoms were present in a very high proportion of our liver transplant population and $75 \%$ of them had moderately severe symptoms. When we compare this group to those at the same center with advanced liver disease, we do not see any significant de- crease in the overall prevalence suggesting that the liver dysfunction is not a direct cause of the symptoms reported. In addition, contrary to the general population and other solid organ transplant recipients RLS was reported more often in men and appeared to be most influenced by the presence of diabetes and hepatitis $C$ and use of medications (antidepressants/antihistamines) known to exacerbate RLS. The prevalence and severity of RLS in this population cannot be overlooked. It can be a significant cause of decreased quality of life that can be easily treated once recognized. A more careful history and examination and understanding of the medication profile which affects the RLS is needed in this group of patients who are already on a number of complex medications.

\section{REFERENCES}

[1] G. J. Lavigne and J. Y. Montplaisir, "Restless Legs Syndrome and Sleep Bruxism: Prevalence and Association among Canadians,” Sleep, Vol. 17, No. 8, 1994, pp. 739743.

[2] D. A. Nichols, R. P. Allen, J. H. Grauke, J. B. Brown, M. L. Rice, P. R. Hyde, W. C. Dement and C. A. Kushida, "Restless Legs Syndrome Symptoms in Primary Care: A Prevalence study," Archives of Internal Medicine, Vol. 163, No. 19, 2003, pp. 2323-2329. doi:10.1001/archinte.163.19.2323

[3] B. Högl, S. Kiechl, J. Willeit, M. Saletu, B. Frauscher, K. Seppi, J. Müller, G. Rungger, A. Gasperi, G. Wenning and W. Poewe, "Restless Legs Syndrome: A CommunityBased Study of Prevalence, Severity, and Risk Factors,” Neurology, Vol. 64, No. 11, 2005, pp. 1920-1924. doi:10.1212/01.WNL.0000163996.64461.A3

[4] G. Merlino, L. Fratticci, M. Valente, A. Del Giudice, C. Noacco, P. Dolso, I. Cancelli, A. Scalise and G. L. Gigli, "Association of Restless Legs Syndrome in Type 2 DIABETES: A Case-Control Study,” Sleep, Vol. 30, No. 7, 2007, pp. 866-871.

[5] J. A. O’Hare, F. Abuaisha and M. Geoghegan, "Prevalence and Forms of Neuropathic Morbidity in 800 Diabetics," Irish Journal of Medical Sciences, Vol. 163, No. 3, 1994, pp. 132-135. doi:10.1007/BF02965972

[6] L. A. Lopes, M. Lins Cde, V. G. Adeodato, D. P. Quental, P. F. de Bruin, R. M. Montenegro Jr. and V. M. de Bruin, "Restless Legs Syndrome and Quality of Sleep in Type 2 Diabetes," Diabetes Care, Vol. 28, No. 11, 2005, pp. 2633-2636. doi:10.2337/diacare.28.11.2633

[7] S. Walker, A. Fine and M. H. Kryger, "Sleep Complaints Are Common in a Dialysis Unit," American Journal of Kidney Diseases, Vol. 26, No. 5, 1995, pp. 751-756. doi:10.1016/0272-6386(95)90438-7

[8] D. Kavanagh, S. Siddiqui and C. C. Geddes, "Restless Legs Syndrome in Patients on Dialysis," American Journal of Kidney Diseases, Vol. 43, No. 5, 2004, pp. 763-771. doi:10.1053/j.ajkd.2004.01.007

[9] S. T. O’Keeffe, K. Gavin and J. N. Lavan, "Iron Status and Restless Legs Syndrome in the Elderly,” Age Ageing, 
Vol. 23, No. 3, 1994, pp. 200-203. doi:10.1093/ageing/23.3.200

[10] C. J. Earley, J. R. Connor, J. L. Beard, E. A. Malecki, D. K. Epstein and R. P. Allen, "Abnormalities in CSF Concentrations of Ferritin and Transferrin in Restless Legs Syndrome,” Neurology, Vol. 54, No. 8, 2000, pp. 16981700. doi:10.1212/WNL.54.8.1698

[11] X. Gao, M. A. Schwarzschild, H. Wang and A. Ascherio, "Obesity and Restless Legs Syndrome in Men and Women,” Neurology, Vol. 72, No. 14, 2009, p. 1255. doi:10.1212/01.wnl.0000345673.35676.1c

[12] Y. A. Rajabally, et al., "Restless Legs Syndrome in Chronic Inflammatory Demyelinating Polyneuropathy,” Muscle \& Nerve, Vol. 42, No. 2, 2010, pp. 252-256. doi:10.1002/mus.21684

[13] S. B. Rutkove, J. K. Matheson and E. L. Logigian, "Restless Legs Syndrome Inpatients with Polyneuropathy," Muscle \& Nerve, Vol. 19, No. 5, 1996, pp. 670-672. doi:10.1002/(SICI)1097-4598(199605)19:5<670::AID-M US20>3.0.CO;2-Q

[14] M I. Botez and B. Lambert, "Folate Deficiency and Restless-Legs Syndrome in Pregnancy,” New England Journal of Medicine, Vol. 297, No. 12, 1977, p. 670. doi:10.1056/NEJM197709222971219

[15] R. A. Franco, "The High Prevalence of Restless Legs Syndrome Symptoms in Liver Disease in an AcademicBased Hepatology Practice,” Journal of Clinical Sleep Medicine, Vol. 4, No. 1, 2008, pp. 45-49.

[16] S. A. Azar, R. Hatefi and M. Talebi, "Evaluation of Effect of Renal Transplantation in Treatment of Restless Legs Syndrome,” Transplantation Proceedings, Vol. 39, No. 4, 2007, pp. 1132-1133. doi:10.1016/j.transproceed.2007.03.097

[17] O. A. Minai, "Restless Legs Syndrome in Lung Transplant Recipients," The Journal of Heart and Lung Transplantation, Vol. 26, No. 1, 2007, pp. 24-29. doi:10.1016/j.healun.2006.10.014

[18] S. Javaheri, "Prevalence of Obstructive Sleep Apnoea and Periodic Limb Movement in 45 Subjects with Heart Transplantation,” European Heart Journal, Vol. 25, No. 3, 2004, pp. 260-266. doi:10.1016/j.ehj.2003.10.032

[19] J. Kirsch, R. P. Allen, T. Washburn and C. J. Earley, "Validation of the Restless Legs Syndrome Quality of Life Questionnaire,” Value in Health, Vol. 8, No. 2, 2005, pp. 157-167. doi:10.1111/j.1524-4733.2005.03010.x

[20] R. P. Allen and C. J. Earley, "Validation of the Johns Hopkins Restless Legs Severity Scale,” Sleep Medicine, Vol. 2, No. 3, 2001, pp. 239-224.

\section{Abbreviations}

RLS: Restless Legs Syndrome;

QoL: Quality of Life;

IRLSSG: International Restless Legs Syndrome Study Group;

IRLS: International RLS Severity Scale Questionnaire;

\section{doi:10.1016/S1389-9457(00)00080-0}

[21] R. P. Allen, C. A. Kushida and M. J. Atkinson, "Factor Analysis of the International Restless Legs Syndrome Study Group's Scale for Restless Legs Severity,” Sleep Medicine, Vol. 4, No. 2, 2003, pp. 133-135. doi:10.1016/S1389-9457(02)00193-4

[22] J. Winkelmann, A. Stautner, W. Samtleben and C. Trenkwalder, "Long-Term Course of Restless Legs Syndrome in Dialysis Patients after Kidney Transplantation," Movement Disorders, Vol. 17, No. 5, 2002, pp. 1072-1076. doi:10.1002/mds.10231

[23] M. Z. Molnar, M. Novak, C. Ambrus, et al., "Restless Legs Syndrome in Patients after Renal Transplantation," American Journal of Kidney Diseases, Vol. 45, No. 2, 2005, pp. 388-396. doi:10.1053/j.ajkd.2004.10.007

[24] T. Kraus, A. Schuld and T. Pollmacher, "Periodic Leg Movements in Sleep and Restless Legs Syndrome Probably Caused by Olanzapine," Journal of Clinical Psychopharmacology, Vol. 19, No. 5, 1999, pp. 478-479. doi:10.1097/00004714-199910000-00017

[25] R. Hargrave and D. J. Beckley, "Restless Legs Syndrome Exacerbated by Sertraline,” Psychosomatics, Vol. 39, No. 2, 1998, pp. 177-178. doi:10.1016/S0033-3182(98)71370-2

[26] R. Bakshi, "Fluoxetine and Restless Legs Syndrome," Journal of the Neurological Sciences, Vol. 142, No. 1-2, 1996, pp. 151-152. doi:10.1016/0022-510X(96)00180-3

[27] M. J. Garvey and G. D. Tollefson, "Occurrence of Myoclonus in Patients Treated with Cyclic Antidepressants," Archives of General Psychiatry, Vol. 44, No. 3, 1987, pp. 269-272. doi:10.1001/archpsyc.1987.01800150081010

[28] T. Terao, M. Terao, R. Yoshimura and K. Abe, "Restless Legs Syndrome Induced by Lithium,” Biological Psychiatry, Vol. 30, No. 11, 1991, pp. 1167-1170. doi:10.1016/0006-3223(91)90185-O

[29] M. S. Aldrich and J. E. Shipley, "Alcohol Use and Periodic Limb Movements of Sleep,” Alcoholism: Clinical \& Experimental Research, Vol. 17, No. 1, 1993, pp. 192 196. doi:10.1111/j.1530-0277.1993.tb00747.x

[30] F. J. Authier, et al., "Detection of Genomic Viral RNA in Nerve and Muscle of Patients with HCV Neuropathy," Neurology, Vol. 60, No. 5, 2003, pp. 808-812. doi:10.1212/01.WNL.0000044399.71601.EA

[31] E. Apartis, "Peripheral Neuropathy Associated with Essential Mixed Cryoglobulinaemia: A Role for Hepatitis C Virus Infection?” Journal of Neurology, Neurosurgery \& Psychiatry, Vol. 60, No. 6, 1996, pp. 661-666. doi:10.1136/jnnp.60.6.661

SD: Standard Deviation;

CI: Confidence Intervals;

SEM: Standard Error of the Mean;

CMV: Cytomegalovirus;

UNOS: United Network of Organ Sharing. 